\title{
gु \\ Mapping the magnetic anisotropy in (Ga,Mn)As nanostructures
}

\author{
F. Hoffmann, G. Woltersdorf, W. Wegscheider, A. Einwanger, D. Weiss, and C. H. Back \\ Department of Physics, Universität Regensburg, 93040 Regensburg, Germany \\ (Received 24 June 2009; revised manuscript received 8 August 2009; published 28 August 2009)
}

\begin{abstract}
Anisotropic strain relaxation in $(\mathrm{Ga}, \mathrm{Mn})$ As nanostructures was studied combining time-resolved Kerr microscopy and ferromagnetic resonance techniques. Local resonance measurements on individual narrow stripes patterned along various crystallographic directions reveal that the easy axis of the magnetization can be forced perpendicular to the strain relaxation direction. Spatially resolved measurements on disk-shaped and rectangular $(\mathrm{Ga}, \mathrm{Mn})$ As structures allow us to directly visualize these local changes in the magnetic anisotropy. We show that the strain-induced edge anisotropy allows for an effective control of the coercive field in stripe structures.
\end{abstract}

PACS number(s): 75.75.+a, 75.30.Gw, 75.50.Pp, 76.50.+g

The magnetic properties of the diluted magnetic semiconductor $(\mathrm{Ga}, \mathrm{Mn})$ As have been studied intensely using ferromagnetic resonance (FMR), superconducting quantum interference device (SQUID) magnetometry, magnetotransport, and Hall-effect measurements due to the potential application of this material in spintronics devices. In order to understand and engineer the magnetotransport properties of such structures it is of utmost importance to understand and control the magnetic anisotropies and the switching behavior. The magnetic anisotropies in $(\mathrm{Ga}, \mathrm{Mn})$ As depend on a multitude of parameters such as temperature, ${ }^{1}$ hole concentration, ${ }^{2}$ and postgrowth annealing. ${ }^{3}$ Recent experiments have shown that the magnetic anisotropies can be manipulated by either applying mechanical stress to the sample ${ }^{4}$ or by releasing the strain-induced anisotropy with lithographic methods..$^{5-7}$ It has also been demonstrated that the easy axis of the magnetization can be rotated by varying the hole concentration using a strong electric field. ${ }^{8}$ However, all these experiments do not resolve magnetic anisotropies in $(\mathrm{Ga}, \mathrm{Mn}) \mathrm{As}$ microand nanostructures locally; the signal in magnetoresistance measurements is always proportional to the average magnetization of the structure.

The experiments presented here allow us to perform spatially resolved measurements of the magnetic anisotropy with a resolution of $500 \mathrm{~nm}$. We have investigated (Ga,Mn)As films grown on GaAs(001) which due to the lattice mismatch grow compressively strained. This strain gives rise to a strong perpendicular magnetic anisotropy with easy axes in the film plane. By patterning a $(\mathrm{Ga}, \mathrm{Mn})$ As film into small structures this strain can be partially relieved, strongly affecting the magnetic anisotropies. Our approach combines the advantages of two experimental techniques: angledependent FMR provides direct access to the energy landscape and the magnetic anisotropies and time-resolved scanning Kerr microscopy allows us to perform time and spatially resolved measurements. Thus these experiments can serve to visualize the local variations in the magnetic anisotropy.

The $\mathrm{Ga}_{1-x} \mathrm{Mn}_{x} \mathrm{As}$ films were grown on GaAs(001) substrates by molecular-beam epitaxy. On the GaAs(001) substrate a 50-nm-thick high-temperature $\mathrm{AlGaAs-buffer} \mathrm{layer}$ and a 8-nm-thick low-temperature GaAs layer were deposited. The subsequent 50-nm-thick $\mathrm{Ga}_{1-x} \mathrm{Mn}_{x} \mathrm{As}$ film with a nominal Mn content of $6 \%(x=0.06)$ was grown at a tem- perature of approximately $510 \mathrm{~K}$. Finally the sample was capped with a thick layer of As. The samples were postgrowth annealed at $470 \mathrm{~K}$ for $20 \mathrm{~h}$ resulting in a Curie temperature of about $110 \mathrm{~K}$ with a hole concentration $p=1.3$ $\times 10^{20} \mathrm{~cm}^{-3}$. In a first step $e$-beam lithography and ionbeam etching was used in order to fabricate the magnetic nanostructures. The etch depth into the GaAs substrate is approximately $30 \mathrm{~nm}$. Stripes having a width ranging from $200 \mathrm{~nm}$ up to $4 \mu \mathrm{m}$ patterned along different crystallographic directions ([100], [110], and [010]) and disks with different diameters were prepared. For the excitation of the magnetization with microwaves in the $\mathrm{GHz}$ range a coplanar waveguide with a bandwidth of $10 \mathrm{GHz}$ was defined in a subsequent lithography step. The magnetic elements are placed in the gap between the 30- $\mu \mathrm{m}$-wide center conductor and the ground plane resulting in a mainly out-of-plane rf excitation [Fig. 1(a)].

Spatially resolved measurements of the magnetic anisotropy in $(\mathrm{Ga}, \mathrm{Mn})$ As nanostructures were performed by using a combination of ferromagnetic resonance and time-resolved scanning Kerr microscopy. Microwaves in the GHz range are synchronized to frequency doubled laser pulses generated by a Ti-sapphire laser with a repetition rate of $80 \mathrm{MHz}$. Similar to conventional FMR measurements the microwave frequency is kept fixed and the magnetic bias field is swept. By changing the phase of the microwaves with respect to the probe pulses both real and imaginary parts of the dynamic susceptibility can be accessed. In our experiment we measure the polar component $M_{z}$ using the magneto-optic Kerr effect. Spatial resolution is obtained by scanning the sample using a piezotable and is limited to about $500 \mathrm{~nm} .^{9,10}$ The high resolution was obtained by using an objective lens with a numerical aperture of 0.6 and a wavelength of $400 \mathrm{~nm}$. The sample is mounted in an optical cryostat. Figure 1(b) shows angle-dependent FMR spectra for a large $60 \times 60 \mu \mathrm{m}^{2}$ (Ga,Mn)As reference sample at $T=7 \mathrm{~K}$ which mimics the response of an isotropically strained unpatterned film. The individual curves are fitted to an asymmetric Lorentz function in order to determine the resonance field.

In order to deduce the anisotropy constants from the FMR measurements it is necessary to derive the resonance condition from the free energy density. ${ }^{1,11}$ In the in-plane geometry both the external magnetic field $H$ and the magnetization 
a)

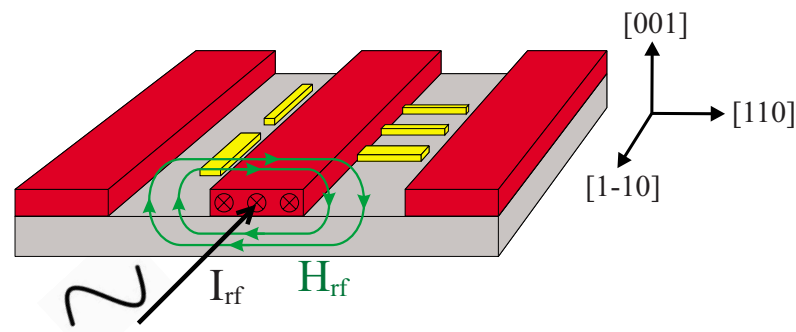

b)

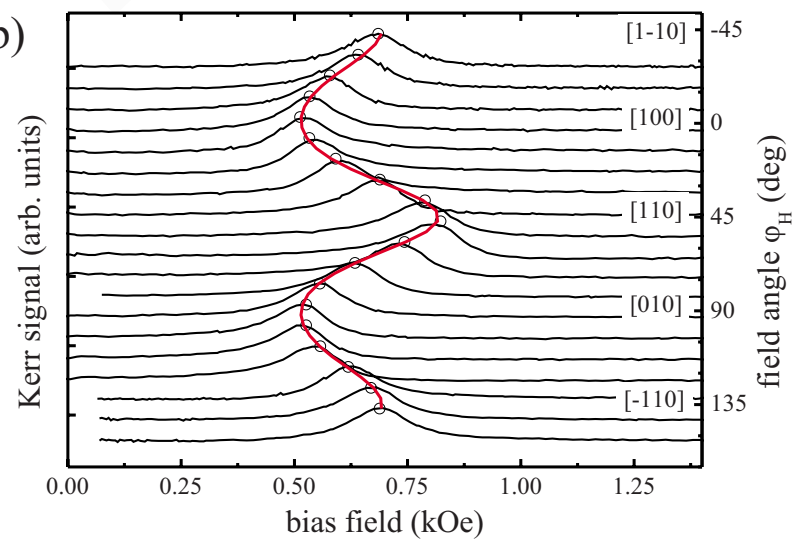

FIG. 1. (Color online) (a) Sketch illustrating the waveguide and the rf excitation of the magnetic elements. (b) FMR spectra for various angles $\varphi_{H}$ of the magnetic field in the film plane (001) for $f=3.84 \mathrm{GHz}$ and $T=7 \mathrm{~K}$ for the reference structure. The resonance fields $H_{R}$ obtained by fitting the experimental data to an asymmetric Lorentz function are given by open dots. The thick red solid line represents the fit which is used to extract the anisotropy constants.

$M$ are aligned in the film plane (001). The free energy density $F$ contains three major contributions: the Zeeman, demagnetizing, and anisotropy energies. (Ga,Ma)As films under compressive strain with a tetragonal distortion show an additional uniaxial in-plane anisotropy and one obtains ${ }^{12}$

$$
\begin{aligned}
F= & -M H\left[\cos \left(\varphi_{\mathrm{M}}-\varphi_{H}\right)\right]-\frac{1}{8} K_{4 \|}\left(3+\cos 4 \varphi_{\mathrm{M}}\right) \\
& -K_{2 \|} \sin ^{2}\left(\varphi_{\mathrm{M}}-\frac{\pi}{4}\right),
\end{aligned}
$$

where $M$ is the magnetization and $\varphi_{\mathrm{M}}$ and $\varphi_{H}$ are the angles of the magnetization and the external magnetic field $H$ with respect to the [100] direction. $K_{2 \|}$ and $K_{4 \|}$ are the uniaxial and cubic in-plane anisotropy constants. The resonance condition is calculated from the Landau-Lifshitz-Gilbert equation and the free energy density resulting in

$$
\left(\frac{\omega}{\gamma}\right)^{2}=B_{\text {eff }} \times H_{\text {eff }}
$$

with

$$
\begin{aligned}
B_{\mathrm{eff}}= & H_{R} \cos \left(\varphi_{\mathrm{M}}-\varphi_{H}\right)+4 \pi M_{\mathrm{eff}}+\frac{K_{4 \|}}{2 M}\left(3+\cos 4 \varphi_{\mathrm{M}}\right) \\
& +\frac{2 K_{2 \|}}{M} \sin ^{2}\left(\varphi_{\mathrm{M}}-\frac{\pi}{4}\right)
\end{aligned}
$$

and

$$
\begin{aligned}
H_{\text {eff }}= & H_{R} \cos \left(\varphi_{\mathrm{M}}-\varphi_{H}\right)+2 \frac{K_{4 \|}}{M} \cos 4 \varphi_{\mathrm{M}} \\
& -\frac{2 K_{2 \|}}{M} \cos \left(2 \varphi_{\mathrm{M}}-\frac{\pi}{2}\right),
\end{aligned}
$$

where $\omega=2 \pi f$ is the angular frequency of the microwave field, $\gamma$ is the gyromagnetic ratio and $4 \pi M_{\mathrm{eff}}=4 \pi M-\frac{K_{2 \perp}}{M}$ is the effective magnetization containing the demagnetization field and the uniaxial perpendicular anisotropy $K_{2 \perp}$ due to the compressive strain.

The angular dependence of Fig. 1(b) (isotropically strained film) is dominated by the intrinsic cubic anisotropy $K_{4}$ due to the symmetry of the zinc-blende lattice. The difference observed along the [110] and [1 10$]$ directions is due to the in-plane uniaxial anisotropy $K_{2 \|}$. The experimental data can be fitted well using the energy density discussed earlier. The resulting anisotropy constants are $K_{4 \|}=2.2$ $\times 10^{3} \mathrm{erg} / \mathrm{cm}^{-3}, K_{2 \|}=-1.1 \times 10^{3} \mathrm{erg} / \mathrm{cm}^{-3}$, and $K_{2 \perp}=-3.0$ $\times 10^{4} \mathrm{erg} / \mathrm{cm}^{-3}$. The magnitude of the perpendicular magnetic anisotropy $K_{2 \perp}$ is comparable to earlier studies with a similar Mn concentration. ${ }^{2,13}$

Next, we address the patterned (Ga,Mn)As structures. Structures with various sizes and shapes were investigated with our local FMR method. First we focus on narrow stripes. The fourfold symmetry of the reference sample shown in Fig. 1(b) changes to a strongly pronounced twofold symmetry when the (Ga,Mn)As film is patterned into a narrow stripe [Fig. 2(a)]. As the lattice can relax partially only along the sides of the stripe for all three orientations of the stripes ([100], [010], and [110]) the easy axis always coincides with the long axis of the stripe; in agreement with earlier studies using SQUID and magnetotransport measurements. ${ }^{5-7}$ One can readily estimate that the shape anisotropy is two orders of magnitude too small to explain the strong uniaxial anisotropy. We determine the size of the strain-relaxation-induced anisotropy $K_{U}$ by simply adding an additional uniaxial anisotropy term $E_{U}=K_{U} \sin ^{2}\left(\varphi_{\mathrm{M}}-\varphi_{U}\right)$ to the free energy density. $\varphi_{U}$ denotes the angle of the easy axis of this additional anisotropy with respect to the [100] direction. By fitting the experimental data for the patterned stripes we derive a value of $E_{U}=1.8 \times 10^{4} \mathrm{erg} / \mathrm{cm}^{-3}$ for the 400 $\mathrm{nm}$-wide stripe which is one order of magnitude stronger than the cubic anisotropy energy of the $(\mathrm{Ga}, \mathrm{Mn})$ As reference sample.

The magnitude of the lattice relaxation for $1-\mu \mathrm{m}$-wide stripes was recently calculated numerically by Wunderlich et $a l .{ }^{5}$ The strain relaxation is spatially nonuniform and takes place on a length scale of several hundred nanometers. ${ }^{5}$ Therefore it is not surprising that we find a very pronounced uniaxial behavior for small stripe widths (400 and $600 \mathrm{~nm}$ ) while for larger widths we observe a mixture of twofold and fourfold symmetries, see Fig. 2(b). We attribute this behavior to the inhomogeneous nature of the lattice relaxation across the stripe width. The data of the wider stripes can be well interpreted as a superposition of fourfold and strainrelaxation-induced twofold symmetry.

The experimentally determined induced uniaxial anisotropy as a function of the reciprocal stripe width is shown in 

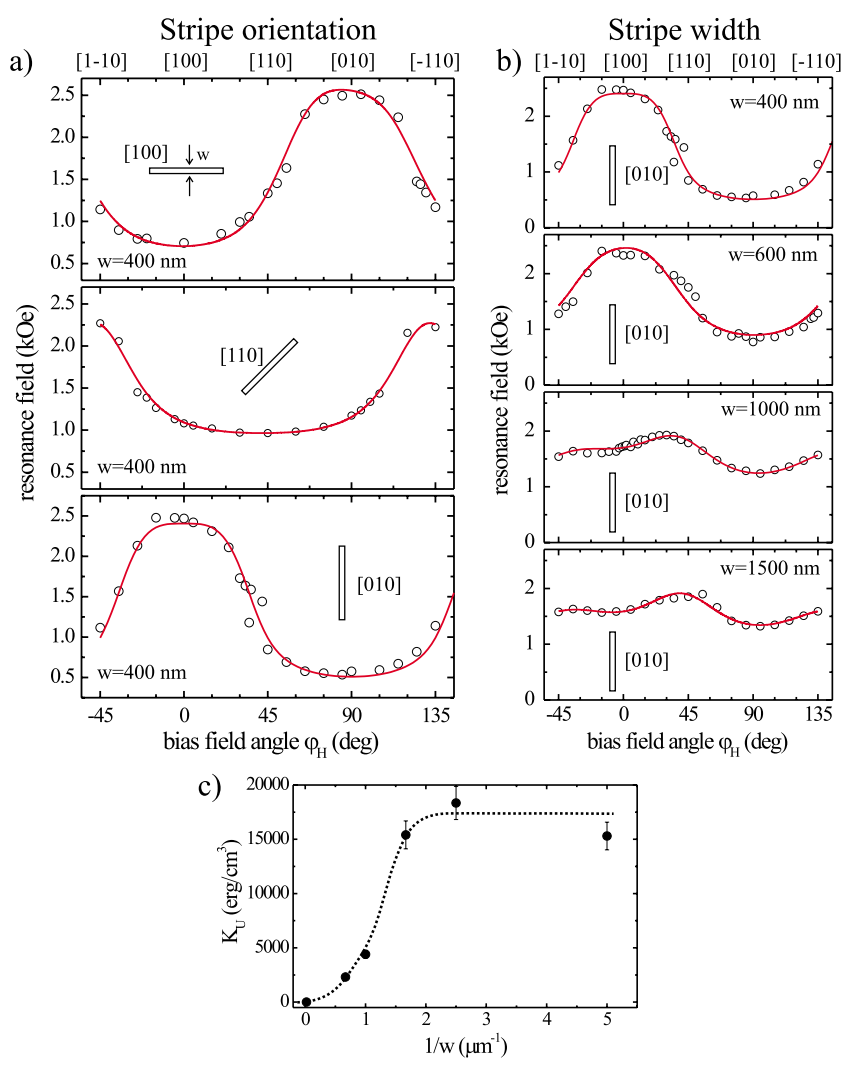

FIG. 2. (Color online) Resonance field as a function of the external field angle $\varphi_{H}$ for stripes patterned along (a) different crystallographic axes and (b) different stripe width $w$. All data were obtained at $f=7.2 \mathrm{GHz}$ and $T=7 \mathrm{~K}$. The solid lines are fits to the experimental data. Panel (c) shows the induced uniaxial anisotropy versus reciprocal stripe width $1 / w$. The dotted line is a guide to the eye.

Fig. 2(c). As expected for large stripe widths, the induced anisotropy approaches zero because the strain-relaxation effects become negligible. For small stripe widths the induced anisotropy saturates. This behavior is a consequence of the complete strain relaxation across the width of the narrowest stripes. These results also demonstrate that the magnitude of the induced uniaxial anisotropy can be well adjusted for stripe widths ranging between 200 and $1500 \mathrm{~nm}$.

In the following we address disk-shaped elements which differ from the rectangular elements in the relaxation direction of the lattice. The angular dependence of the resonance fields for a disk with a large diameter $(d=10 \mu \mathrm{m}$, black symbols) and a small diameter $(d=1.5 \mu \mathrm{m}$, open symbols $)$ is shown in Fig. 4(a). The experimental data for the 10 - $\mu \mathrm{m}$-diameter disk were obtained at the disk center where the strain relaxation is almost zero. We want to emphasize that for a narrow rectangular structure the strain can only relax in one direction giving rise to a strong uniaxial anisotropy [Fig. 2(a)]. For a disk-shaped element we expect isotropic strain relaxation. For this reason the in-plane anisotropies $K_{2 \|}$ and $K_{4 \|}$ should remain constant while the straininduced perpendicular anisotropy $K_{2 \perp}$ should be reduced. This reduction is indeed observed [Fig. 4(a)] as the resonance fields are only shifted to higher field values. The fits to the experimental data show that $K_{2 \|}$ and $K_{4 \|}$ remain almost

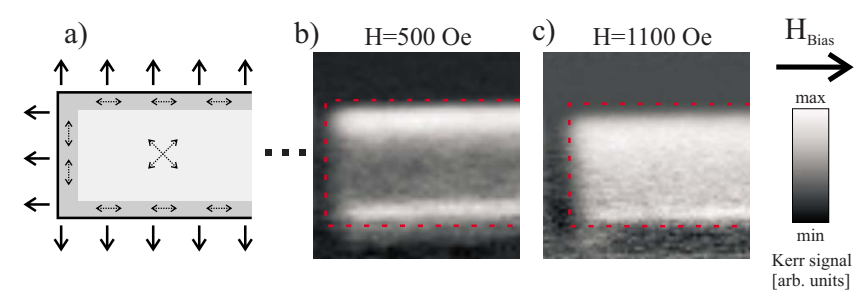

FIG. 3. (Color online) (a) Sketch illustrating the strainrelaxation direction (bold arrows) and the local easy axes (thin arrows) for a large rectangular structure. [(b) and (c)] Images of the polar Kerr signal for a large rectangular structure $(60 \mu \mathrm{m}$ $\times 14 \mu \mathrm{m})$ for $f=4.6 \mathrm{GHz}$. The red dotted lines indicate the outer boundaries of the elements.

constant while $K_{2 \perp}$ is reduced by approximately $20 \%$ for the disk with $1.5 \mu \mathrm{m}$ diameter.

Finally we demonstrate the ability to visualize the local variations in the magnetic anisotropies for both the diskshaped and the rectangular $(\mathrm{Ga}, \mathrm{Mn})$ As structures. Figures 3(b), 3(c), and 4(c)-4(e) show spatially resolved images of the Kerr signal obtained at a fixed excitation frequency and applied magnetic field.

When comparing the circular and rectangular geometries one finds laterally isotropic and anisotropic strain relaxations, respectively [indicated by the thick arrows in Figs. 3(a) and 4(b)]. Furthermore for larger structures one can distinguish two regions as illustrated in the figures: region 1 located at the boundary of the structure where the strain relaxation takes place and a strong strain-induced magnetic anisotropy can be expected, and region 2 in the center of the structure where the $(\mathrm{Ga}, \mathrm{Mn})$ As lattice is fully strained and the magnetic anisotropy is unchanged compared to the extended $(\mathrm{Ga}, \mathrm{Mn})$ As film. As a consequence the magnetic anisotropies and the direction of the easy axes of the magne-
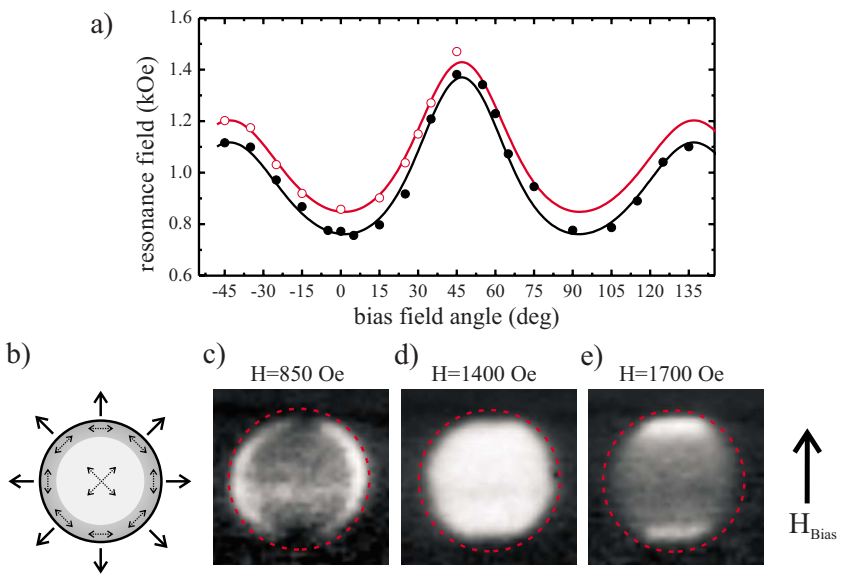

FIG. 4. (Color online) (a) Angular dependence of the resonance field for disk-shaped (Ga,Mn)As elements for $f=5.8 \mathrm{GHz}$ and $T$ $=7 \mathrm{~K}$. The full black and the open red dots represent the experimental data for a disk with a diameter $d=10$ and $1.5 \mu \mathrm{m}$, respectively. The solid lines are obtained by fitting the experimental data. (b) Illustrates the strain relaxation in and the associated distribution of anisotropies in a circular structure. [(c)-(e)] Images of the polar Kerr signal for a disk-shaped element with a diameter of $10 \mu \mathrm{m}$ for $f=5.8 \mathrm{GHz}$. 
tization are varying locally. Based on previous results we expect the local easy axes (shown by the thin arrows) to be parallel to the boundary of the elements, whereas in the central region we expect the film like behavior with easy axes along the $\langle 100\rangle$ directions.

Spatially nonuniform magnetic anisotropies lead to a nonuniform magnetic response for uniform excitation. In particular, the condition for FMR is only satisfied locally. This behavior is clearly observed in Figs. 3(b), 4(c), and 4(e). For the rectangular structure the Kerr signal shows two stripes parallel to the external field direction for $H=500 \mathrm{Oe}$ whereas for $H=1100$ Oe the magnetic response is almost uniform. The strain relaxation at the boundary induces a uniaxial anisotropy with an easy axis parallel to the stripe thus lowering the ferromagnetic resonance field. A similar behavior is also found for the circular structure (see Fig. 4). Indeed at the resonance field of the disk center [which for $\varphi_{H}=45^{\circ}$ is approximately $H_{U}=1400$ Oe, see Fig. 4(a)] the magnetic response is uniform [Fig. 4(d)]. However for $H$ $<H_{U}$ two ring-shaped modes at the disk boundary [Fig. 4(c)] and for $H>H_{U}$ two modes localized at the edges are found [Fig. 4(e)]. Note that for $H<H_{U}$ these ring-shaped modes are aligned along the field direction while for $H>H_{U}$ the modes are aligned perpendicular to $H$. The ring-shaped modes can be explained in the following way: due to the lattice relaxation at the disk boundary the easy axis follows the circumference. The results for narrow stripes have shown that a parallel alignment of the magnetization with respect to the long axis of the stripe is favored. Therefore we expect for the circular structures that the local easy axis is aligned tangentially with respect to the curved boundary. For the ringshaped areas the external field is aligned along the local easy axes which means that the resonance is observed at a lower bias field than the main resonance. In contrast for the areas located at the bottom and top of the image the bias field is aligned along a local hard axis, which results in a higher resonance field. These findings are consistent with other measurements where the external field was applied along different directions (not shown here).

The inhomogeneous magnetic anisotropies also have a severe effect on the static magnetic properties of the elements such as the magnetic ground state and the coercive field. This is illustrated for a 400-nm-wide stripe in Fig. 5. Experimentally we find a coercivity of only 50 Oe while the StonerWohlfarth model and micromagnetic simulations predict a coercive field of 800 Oe. When the strain-induced edge anisotropies that we determined by our local FMR measurements are included in the micromagnetic simulation we find that the noncollinear edge regions nucleate the reversal leading to a more than $50 \%$ reduced coercivity of 400 Oe compared to the simulation with uniform magnetic properties [see Fig. 5(d) for a map of the local anisotropy that was used in the simulation and the corresponding magnetic ground state]. The even lower coercive field of 50 Oe that is measured in the experiment is probably a consequence of thermal excitations that are not considered in the simulation. The temperature in the experiment is only $6 \mathrm{~K}$ but in (Ga,Mn)As the spin-wave parameter is more than two orders of magnitude larger than, e.g., in iron. ${ }^{14}$ The large spin-wave parameter in $(\mathrm{Ga}, \mathrm{Mn}) \mathrm{As}$ can be attributed to a large enhancement

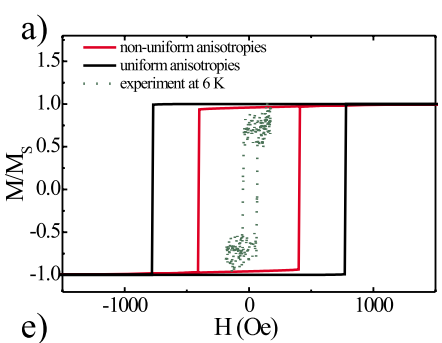

b)
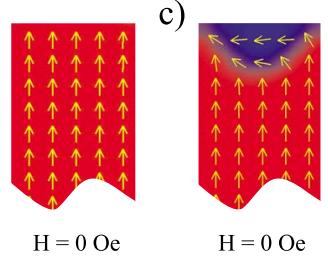

d)
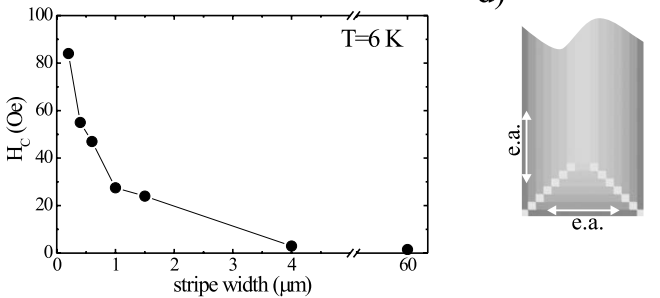

$\mathrm{K}_{\mathrm{U}}\left(10^{4} \mathrm{erg} / \mathrm{cm}^{3}\right)$

$\begin{array}{lll} & 1.0 \quad 3.2\end{array}$
FIG. 5. (Color online) (a) Hysteresis loops obtained by micromagnetic simulations for a 400-nm-wide and 4- $\mu \mathrm{m}$-long stripe with spatially uniform magnetic anisotropies (black line) and local magnetic anisotropies (red line). The external field was applied parallel to the long axis of the stripe. The dotted line shows experimental data measured on a corresponding structure at $6 \mathrm{~K}$. (b) Magnetic ground state calculated using uniform (averaged) magnetic anisotropies. (c) Modified magnetic ground state due to inhomogeneous magnetic anisotropies. (d) Local distribution of the uniaxial magnetic anisotropy used for the calculation of the ground state (c) and the hysteresis loop (red line) in (a). The directions of the local easy axes are given by the arrows. (e) Experimentally determined coercive fields as a function of stripe width $w$.

of the density of low-energy magnetic excitations due to disorder. This strong magnon softening is related to the reduced bulk spin couplings which are caused by a preferential accumulation of holes in spin clusters. This accumulation leads to a depletion of holes from the bulk spins and thereby enhances the low-energy extended-state magnon modes. ${ }^{15} \mathrm{Al}$ though the switching field is largely reduced due to thermally excited spin waves the anisotropic strain relaxation still allows to control the switching field by varying the width of stripe structures. As can be seen in Fig. 5(e) the coercive field changes from 1.5 Oe for the extended film to about 80 Oe for 200-nm-wide stripes. In addition the presence of a uniaxial anisotropy with the magnetic easy axis parallel to the edges also leads to a modified magnetic ground state, Fig. 5(c). The observed behavior is very important for transport measurements with $(\mathrm{Ga}, \mathrm{Mn})$ As electrodes for two reasons: the noncollinear magnetic ground state reduces the measured magnetoresistive signal. More importantly the control over the coercivity by varying stripe width allows to engineer well defined and independent switching of injector and detector electrodes, see Fig. 5(e).

In conclusion, we have investigated the effects of strain relaxation on the magnetic anisotropies in (Ga,Mn)As structures by means of local ferromagnetic resonance measurements. In rectangular structures with anisotropic strain relaxation a strong induced uniaxial magnetic in-plane anisotropy is found with an easy axis perpendicular to the strain- 
relaxation direction. These results were supported by our spatially resolved measurements allowing us to directly visualize local variations in the magnetic anisotropy occurring on a submicrometer scale. Our results show that the magnetic anisotropies and the coercive field in (Ga,Mn)As can be well controlled by lithography. In metallic ferromagnets with large magnetization dipolar (demagnetizing) effects can lead to similar phenomena. However here we have shown that strain relaxation can mimic these effects in a material with a very small magnetization leading to edge localized resonance modes and shape-induced magnetic anisotropy.

Financial support from the German Research Foundation through SFB 689 is gratefully acknowledged.
${ }^{1}$ X. Liu, Y. Sasaki, and J. K. Furdyna, Phys. Rev. B 67, 205204 (2003).

${ }^{2}$ K. Khazen, H. J. von Bardeleben, J. L. Cantin, L. Thevenard, L. Largeau, O. Mauguin, and A. Lemaître, Phys. Rev. B 77, 165204 (2008).

${ }^{3}$ V. Stanciu and P. Svedlindh, Appl. Phys. Lett. 87, 242509 (2005).

${ }^{4}$ C. Bihler, M. Althammer, A. Brandlmaier, S. Geprägs, M. Weiler, M. Opel, W. Schoch, W. Limmer, R. Gross, M. S. Brandt, and S. T. B. Goennenwein, Phys. Rev. B 78, 045203 (2008).

${ }^{5}$ J. Wunderlich et al., Phys. Rev. B 76, 054424 (2007).

${ }^{6}$ J. Wenisch, C. Gould, L. Ebel, J. Storz, K. Pappert, M. J. Schmidt, C. Kumpf, G. Schmidt, K. Brunner, and L. W. Molenkamp, Phys. Rev. Lett. 99, 077201 (2007).

${ }^{7}$ S. Hümpfner, K. Pappert, J. Wenisch, K. Brunner, C. Gould, G. Schmidt, L. W. Molenkamp, M. Sawicki, and T. Dietl, Appl. Phys. Lett. 90, 102102 (2007).
${ }^{8}$ D. Chiba, M. Sawicki, Y. Nishitani, Y. Nakatani, F. Matsukura, and H. Ohno, Nature (London) 455, 515 (2008).

${ }^{9}$ G. Woltersdorf, O. Mosendz, B. Heinrich, and C. H. Back, Phys. Rev. Lett. 99, 246603 (2007).

${ }^{10}$ I. Neudecker, K. Perzlmaier, F. Hoffmann, G. Woltersdorf, M. Buess, D. Weiss, and C. H. Back, Phys. Rev. B 73, 134426 (2006).

${ }^{11}$ X. Liu and J. K. Furdyna, J. Phys.: Condens. Matter 18, R245 (2006).

${ }^{12}$ M. Farle, Rep. Prog. Phys. 61, 755 (1998).

${ }^{13}$ S. T. B. Goennenwein, T. Graf, T. Wassner, M. S. Brandt, M. Stutzmann, J. B. Philipp, R. Gross, M. Krieger, K. Zürn, P. Ziemann, A. Koeder, S. Frank, W. Schoch, and A. Waag, Appl. Phys. Lett. 82, 730 (2003).

${ }^{14}$ M. Sperl, A. Singh, U. Wurstbauer, S. K. Das, A. Sharma, M. Hirmer, W. Nolting, C. H. Back, W. Wegscheider, and G. Bayreuther, Phys. Rev. B 77, 125212 (2008).

${ }^{15}$ A. Singh, Phys. Rev. B 75, 035206 (2007). 